\title{
Chimera states in nonlocally coupled phase oscillators with biharmonic interaction
}

\author{
Hongyan Cheng, Qionglin Dai, Nianping Wu, Yuee Feng, \\ Haihong Li, and Junzhong Yang* \\ School of Science, Beijing University of Posts and Telecommunications, Beijing, \\ 100876, People's Republic of China
}

E-mail: jzyang@bupt.edu.cn

\begin{abstract}
Chimera states, which consist of coexisting domains of coherent and incoherent parts, have been observed in a variety of systems. Most of previous works on chimera states have taken into account specific form of interaction between oscillators, for example sinusoidal coupling or diffusive coupling. Here, we investigate chimera dynamics in nonlocally coupled phase oscillators with biharmonic interaction. We find novel chimera states with features such as that oscillators in the same coherent cluster may split into two groups with a phase difference between them at around $\pi / 2$ and that oscillators in adjacent coherent clusters may have a phase difference close to $\pi / 2$. The different impacts of the coupling ranges in the first and the second harmonic interactions on chimera dynamics are investigated based on the synchronous dynamics in globally coupled phase oscillators. Our study suggests a new direction in the field of chimera dynamics.

PACS numbers: 05.45.Xt, 89.75.kd
\end{abstract}




\section{Introduction}

Chimera states refer to a type of spatiotemporal pattern in which identical oscillators spontaneously split into coexisting and spatially separated domains with dramatically different behaviors, i.e., coherent and incoherent oscillations. Chimera states were first numerically found in a ring of nonlocally coupled Ginzburg-Landau oscillators [1]. Later, Abrams and Strogatz presented theoretical results for the states in a ring of phase oscillators coupled by a cosine kernel [2]. Chimera states have been studied intensively over the past years [3, 4, [5, 6, 17, 8, 9, 10, 11, 12, 13, 14, 15]. They have been found in the systems with different topologies, such as square lattices [16, 17, 18], torus [19], and complex networks [20]. The systems in which chimera states are observed include timediscrete maps [21], time-continuous chaotic models [22], neural systems [23, 24, 25], and so on. Recently, chimera states have been realized experimentally in optical [26, 27], chemical [28, 29], mechanical and electronic systems [30, 31, 32].

Generally, the system of nonlocally coupled identical oscillators on a ring with length $L$ can be described as

$$
\dot{\mathbf{u}}(x)=\mathbf{f}[\mathbf{u}(x)]+\epsilon \int_{0}^{L} G\left(x-x^{\prime}\right) \mathbf{H}\left[\mathbf{u}(x)-\mathbf{u}\left(x^{\prime}\right)\right] d x^{\prime} .
$$

The kernel functions widely used are exponential function $G(x)=e^{-\kappa|x|}$ and step function $G\left(x-x^{\prime}\right)=1$ if $\left|x-x^{\prime}\right| \leq \sigma$ or $G\left(x-x^{\prime}\right)=0$ otherwise, where $\kappa$ and $\sigma$ measure the coupling range. For phase oscillators, $u(x)=\theta(x)$, the phase of oscillator at position $x$, is a scalar variable and $f[u(x)]=\omega$ the natural frequency of oscillators which can be set to be zero without the loss of generality. In previous investigations on nonlocally coupled oscillators, the interaction among oscillators always takes the form of diffusion coupling which manifests itself as $\sin \left[\theta(x)-\theta\left(x^{\prime}\right)+\alpha\right]$, the first order of $H$ in the Fourier expansion, for phase oscillators. However, in more general cases, $H$ should be approximated by a biharmonic coupling function $\epsilon_{1} \sin \left[\theta(x)-\theta\left(x^{\prime}\right)+\alpha_{1}\right]+\epsilon_{2} \sin 2\left[\theta(x)-\theta\left(x^{\prime}\right)+\alpha_{2}\right]$ [33, 34, 35, 36]. In fact, sinusoidally coupled systems are typically degenerate, so the inclusion of the second harmonic interaction may lead to more generic bifurcation behaviors.

In this paper, We investigate nonlocally coupled phase oscillators with biharmonic interaction. Especially, we suppose that the first harmonic and the second harmonic interactions may have different coupling ranges. We show the existence of novel chimera states with the features resulted from the biharmonic interaction. We explore the impacts of the coupling ranges in the first and the second harmonic interactions on chimera states. We present the mechanism for these novel chimera states based on the synchronization in globally coupled phase oscillators. 


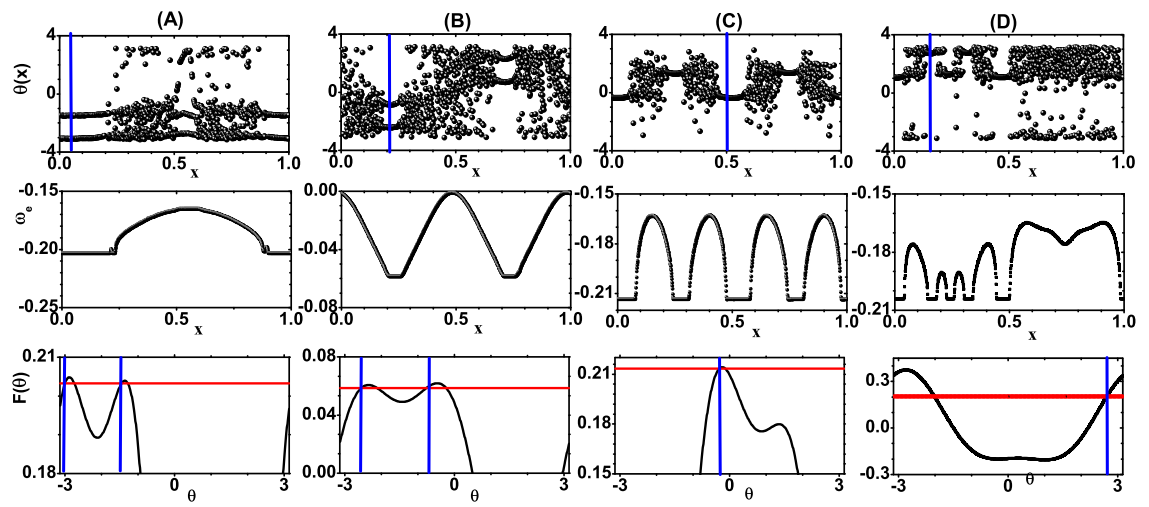

Figure 1. (color online) Examples of typical chimera states in the model Eq. (2). (A) The C1P2 chimera state at $\kappa_{1}=4$ and $\kappa_{2}=4$ (B) The C2P2 chimera state at $\kappa_{1}=2$ and $\kappa_{2}=4$. (C) The C4P1 chimera state at $\kappa_{1}=4$ and $\kappa_{2}=0.5$. (D) The C5P1 chimera state at $\kappa_{1}=4$ and $\kappa_{2}=1.4$. The top panels show the snapshots $\theta(x)$ and the middle panels show the corresponding effective frequencies $\omega_{e}(x)$. In the bottom panels, the black curve plots the function $F(\theta)$ where the order parameters $Z_{1,2}$ are acquired from the oscillator at the position denoted by the blue line in the top panel and the red line plots $-\Omega$ the effective frequency in the coherent clusters. The blue lines in the bottom panel denote the stable solutions $\theta(x)$ to Eq. (6). $N=1000$.

\section{Model}

We consider a ring of identical phase oscillators with nonlocal coupling with fixed length $L=1$. The equation of motion for the system is described as

$$
\begin{aligned}
\dot{\theta}(x) & =-\sum_{m=1,2} \epsilon_{m} \int_{0}^{1} G_{m}\left(x-x^{\prime}\right) \\
& \times \sin m\left[\theta(x)-\theta\left(x^{\prime}\right)+\alpha_{m}\right] d x^{\prime}
\end{aligned}
$$

Here, the angles $\alpha_{1}$ and $\alpha_{2}$ are tunable parameters that describe the phase shifts between oscillators at $x$ and $x^{\prime}$ in the first and the second harmonic interactions, respectively. $\epsilon_{1}$ and $\epsilon_{2}$ describe the corresponding coupling strengthes. The kernel functions $G_{1,2}$, decaying exponentially with the distance between the oscillators, take the form

$$
G_{1,2}(x)=e^{-\kappa_{1,2}|x|} .
$$

$\kappa_{1}$ and $\kappa_{2}$ measure the coupling ranges in the first and the second harmonic interactions. To be noted, we do not normalize the kernel functions to have unit integral and, actually, the normalization constants are absorbed into the coupling strengthes.

We define two position-dependent complex order parameters (generalized Daido order parameters [39, 40]) $Z_{m}(x)=R_{m}(x) e^{i m \Theta_{m}(x)}$ with $m=1,2$ as

$$
R_{m}(x) e^{i m \Theta_{m}(x)}=\int_{0}^{1} G_{m}\left(x-x^{\prime}\right) e^{i m \theta\left(x^{\prime}\right)} d x^{\prime} .
$$


Then Eq. (2) becomes

$$
\dot{\theta}(x)=-\sum_{m=1}^{2} \epsilon_{m} R_{m}(x) \sin m\left[\theta(x)-\Theta_{m}(x)+\alpha_{m}\right]
$$

Now, the mutual entrainment among oscillators depends on both $Z_{1}$ and $Z_{2}$.

Before going further, there are several remarks on the model. Firstly, the model at $\kappa_{1}=0$ and $\kappa_{2}=0$ is reduced to a globally coupled one. It has been investigated recently for nonidentical phase oscillators with $\alpha_{1,2}=0$, where the presence of the second harmonic interaction term leads to an infinite number of coherent states [37, 38. On the other hand, the model with the limit $\kappa_{1} \rightarrow \infty$ and $\kappa_{2} \rightarrow \infty$ represents the locally coupled one. Secondly, both the model with $\epsilon_{1} \neq 0$ and $\epsilon_{2}=0$ and the model with $\epsilon_{1}=0$ and $\epsilon_{2} \neq 0$ support chimera states. In these two cases, chimera states coexist with the synchronous state and realizing a chimera state requires special initial conditions. There are an infinite number of chimera states in the case of $\epsilon_{1}=0$ and $\epsilon_{2} \neq 0$ due to the invariance of the model under the transformation $\theta(x) \rightarrow \theta(x)+\pi$ for any $x$. Thirdly, a similar version of the model Eq. (2) has been studied by Suda and Okuda where the kernel function is a step function [41]. They considered the situation with $\epsilon_{2} \ll \epsilon_{1}$ and found two critical $\epsilon_{2}$. At the first critical $\epsilon_{2}$, the synchronous state becomes unstable and, then, chimera states may live forever. Beyond the second critical $\epsilon_{2}$, chimera states are unstable. In this paper, we will show that chimera states can exist for larger $\epsilon_{2}$ and these states display novel features induced by the second harmonic interaction. Ashwin and his collaborators studied the model with biharmonic interaction in small systems and proposed the concept of weak chimeras [42, 43, 44].

\section{Results}

We numerically simulate the model using a fourth-order Runge-Kutta method with time step $\delta t=0.05$. The results are examined by shorter time step such as $\delta t=0.01$. The ring is discretized into $N$ oscillators with $N=256$ or $N=1000$. Throughout the paper, $\alpha_{1}=1.45$ and $\alpha_{2}=1.45$. Without loss of generality, we set $\epsilon_{1}=1$. We first let $\epsilon_{2}=4$ to show the phenomenology of chimera states unique to the model with the biharmonic interaction and to investigate their dependence on the coupling ranges $\kappa_{1}$ and $\kappa_{2}$. Then we present the dependence of the critical $\epsilon_{2}$ on $\kappa_{2}$ above which these chimera states exist.

\subsection{Typical chimera states}

We focus on the parameter regime $\left(\kappa_{1}, \kappa_{2}\right) \in[0,4] \times[0,4]$. In most part of the regime, chimera states are stable provided that $\kappa_{1}$ is not too close to zero, and can be realized with random initial conditions where $\theta(x)$ is randomly drawn from $[0,2 \pi]$. There exist many types of chimera states. Some typical chimera states are presented in Fig. 1 and discussed as follows. 

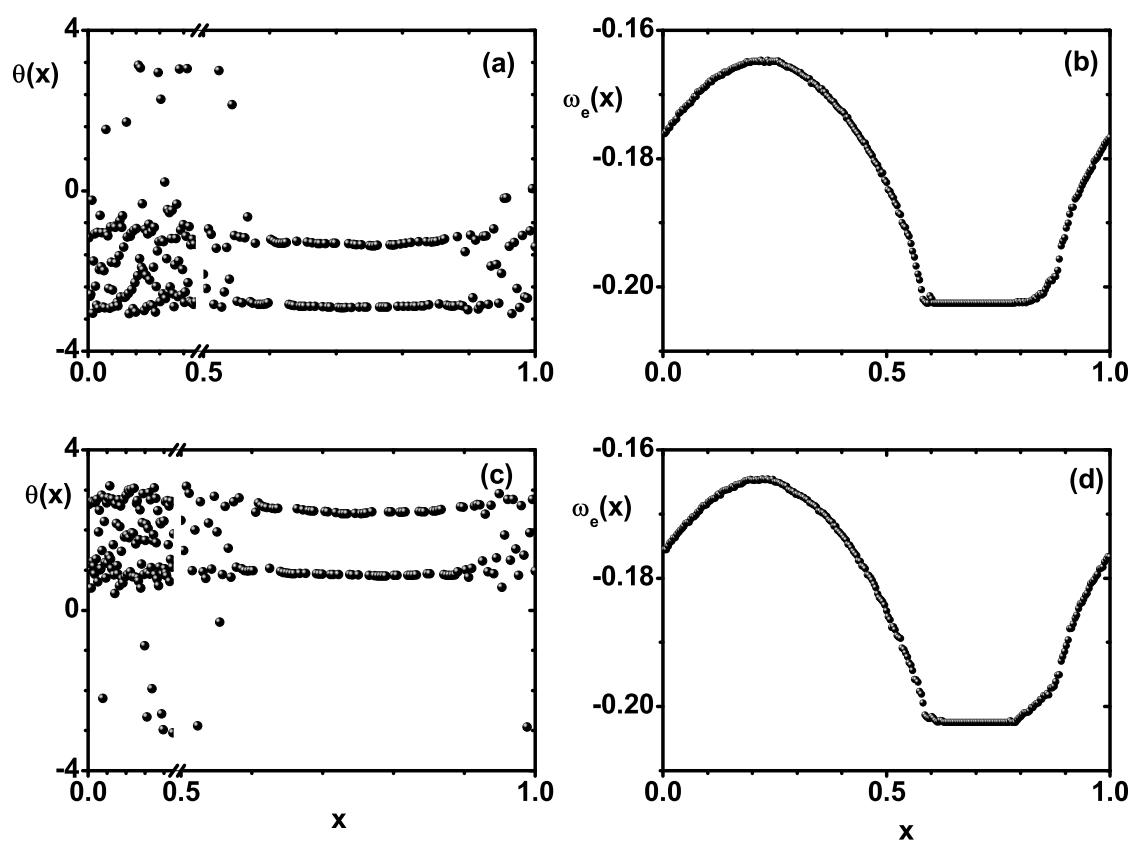

Figure 2. (color online) Two C1P2 chimera states at $\kappa_{1}=4$ and $\kappa_{2}=4$ generated from different random initial conditions. $N=256$. (a) and (c) The snapshots $\theta(x)$; (b) and (d) The corresponding effective frequencies $\omega_{e}(x)$. Though both $\theta(x)$ and the profiles of $\omega_{e}(x)$ for these two chimera states look the same, the partitions of oscillators into two groups in the coherent cluster are different from each other.

1) C1P2 chimera states A chimera state at $\kappa_{1}=4$ and $\kappa_{2}=4$ is presented in Fig. 1(A) where the snapshot $\theta(x)$ and the effective frequencies $\omega_{e}(x)$ of oscillators are plotted in the top and the middle panels, respectively. Here, the effective frequency $\omega_{e}(x)$ is defined as $\omega_{e}(x)=\left\langle\frac{d \theta(x)}{d t}\right\rangle_{t}$ with $\langle\cdot\rangle_{t}$ the average over time. The graph $\omega_{e}(x)$ shows clearly that oscillators split into two clusters, one coherent cluster in which oscillators share the same effective frequency and one incoherent cluster in which oscillators have different effective frequencies. Different $\omega_{e}(x)$ leads to scattered oscillators in the incoherent cluster. However, different from the chimera states in previous investigations where oscillators in the same coherent cluster are nearly in phase, Fig. 1(A) shows that the same $\omega_{e}(x)$ does not lead oscillators to be nearly in phase. As indicated by two nearly horizontal lines in the coherent cluster in the graph $\theta(x)$, oscillators in the coherent cluster are divided into two groups: oscillators in the same group are nearly in phase and those in different groups have a phase difference between them at around $\pi / 2$. In the following, we denote as $\mathrm{CnPm}$ a chimera state with $n$ coherent clusters and $m$ groups of oscillators with different phases in a same coherent cluster is Thus, the chimera state in Fig. 1(A) is a C1P2 state.

The $\mathrm{P} 2$ phenomenon in a coherent cluster results from the biharmonic interaction. To be clear, we consider oscillators in the coherent cluster. For these oscillators, their 



Figure 3. (color online) The forward and backward transition diagrams between the $\mathrm{C} 2 \mathrm{P} 1$ and $\mathrm{C} 4 \mathrm{P} 1$ chimeras are presented in (a) and (b) by monitoring $\sigma(x)$, respectively. In these plots, coherent clusters are represented by black color where $\sigma(x)=0$. (c), (d) and (e) show the snapshots $\theta(x)$ at $\kappa_{2}$ denoted by the red arrows. The forward and backward transition diagrams between the $\mathrm{C} 4 \mathrm{P} 1$ and $\mathrm{C} 8 \mathrm{P} 1$ chimeras are presented in (f) and (g), respectively. (h), (i) and (j) show the snapshots $\theta(x)$ at $\kappa_{2}$ denoted by the red arrows. $\kappa_{1}=4$ and $N=256$.

phases are locked to the mean fields and the phase differences $\theta(x)-\Theta_{1,2}(x)$ between theirs and the mean fields can be obtained by looking for the stable equilibria in Eq. (5). By using $\dot{\theta}(x)=\Omega$ for these oscillators, Eq. (5) yields

$$
-\Omega=F\left[\theta^{*}(x)\right]
$$

with $F[\theta(x)]=\sum_{m=1,2} \epsilon_{m} R_{m}(x) \sin m\left[\theta(x)-\Theta_{m}(x)+\alpha_{m}\right]$. The phase differences $\theta^{*}(x)-\Theta_{1,2}(x)$ between these oscillators and the mean fields $Z_{1,2}$ can be obtained by solving Eq. (6) [To be noted, both $\theta^{*}(x)$ and $\Theta_{1,2}(x)$ are time-dependent]. For $\epsilon_{2}=0$, Eq. (6) has two solutions and only one of them is stable. Therefore, there is only one phase which can be taken by an oscillator in the coherent cluster at any time, which is the reason behind that oscillators in the coherent cluster stay nearly in phase and is what we have observed in chimera states previously investigated. However, for the biharmonic interaction with $\epsilon_{2} \neq 0$, it is likely that there are four different $\theta^{*}(x)-\Theta_{1,2}(x)$ satisfying Eq. (6) and two of them are stable equilibria to Eq. (5), which means that there are two different phases to be taken by an oscillator in the coherent cluster. Consequently, oscillators in the coherent cluster spilt into two groups and oscillators in different groups fall onto different equilibria. The possible bistability in Eq. (5) provides 


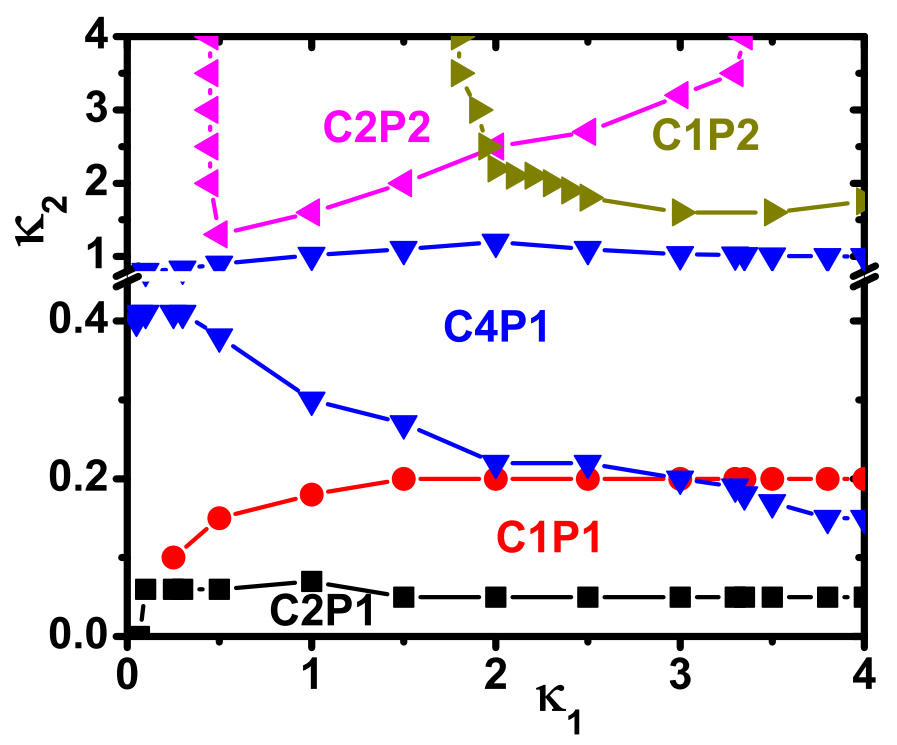

Figure 4. (color online) Stability regimes for several typical chimera states in the plane of $\kappa_{1}$ and $\kappa_{2}$. The C2P2 and C1P2 states are stable above the magenta and dark yellow curves, respectively. The stable C4P1 is enclosed by the blue curves. The C1P1 state is stable between the red and black curves while the $\mathrm{C} 2 \mathrm{P} 1$ state is stable below the black curve. $N=256$.

an explanation on the P2 phenomenon. That the bistability does exist in the chimera state in Fig. 1(A) is examined in the bottom panel where we consider an oscillator whose location is indicated by the blue line in the top panel. $F(\theta)$ against $\theta$ for the oscillator is presented with $Z_{1,2}$ and $\Omega$ acquired in the simulation. As shown in the plot, there exist four solutions and two of them, denoted by blue lines, are stable. Comparing with the top panel, we find that two stable $\theta^{*}(x)$ are the same as the phases taken by the oscillator.

In addition, which group an oscillator in the coherent cluster belongs to depends on initial conditions. Therefore, the oscillators in the coherent cluster randomly distribute themselves into these two groups. For different initial conditions, the 2-group partitions are different. For example, we present in Fig. 2 two C1P2 states at $\kappa_{1}=4$ and $\kappa_{2}=4$ with different initial conditions. The snapshots $\theta(x)$ show different partitions though their graphs $\omega_{e}(x)$ look the same. If different partitions of oscillators in the coherent cluster refer to different chimera states, there are a large number of C1P2 states.

2) C2P2 chimera states. As shown in Fig. 1(B), the state at $\kappa_{1}=2$ and $\kappa_{2}=4$ consists of two coherent clusters separated by incoherent ones. In each coherent cluster, oscillators split into two groups with a phase difference between them at around $\pi / 2$. Moreover, the oscillators in different coherent clusters have a phase difference at around $\pi$. The two coherent clusters always have the same size. Except for the two groups of oscillators with different phases in each coherent cluster, a C2P2 state looks similar to 
the clustered chimera states in delay-coupled phase oscillators without the biharminic interaction [45].

3) Multi-cluster chimera states with translation symmetry. This type of chimera states always manifest themselves as $\mathrm{C} 2 \mathrm{nP} 1$ or $\mathrm{CnP} 1$ states and have a spatial period $1 / n$. A typical C2nP1 state consists of $2 n$ coherent clusters interspersed by $2 n$ incoherent ones and adjacent coherent clusters always have different sizes. In each coherent cluster, oscillators are nearly in phase. Different from previously studied multi-cluster chimera states [46, 47], there is a phase difference at around $\pi / 2$ between the adjacent coherent clusters instead of $\pi$. In Fig. 1(C), we show a C2nP1 state with $n=2$ at $\kappa_{1}=4$ and $\kappa_{2}=0.5$. As indicated by the bottom panel, there is only one stable equilibrium to Eq. (5) for oscillators in coherent clusters. On the other hand, a typical CnP1 chimera state with spatial period $1 / n$ consists of $n$ coherent clusters interspersed by $n$ incoherent ones. In $\mathrm{CnP} 1$ chimera states, adjacent coherent clusters always have the same size and are nearly in phase between them. As shown later, $\mathrm{CnP} 1$ chimera states with spatial period $1 / n$ have close relation with $\mathrm{C} 2 \mathrm{nP} 1$ states.

4) Multi-cluster chimera states with reflection symmetry. This types of chimera states consist of $\mathrm{C} 2 \mathrm{n}+1 \mathrm{P} 1$ and $\mathrm{C} 2 \mathrm{n}+1 \mathrm{P} 2$ states. In the states, coherent clusters always have different sizes and adjacent coherent clusters have a phase difference at $\pi / 2$. The states own one largest incoherent cluster whose size may be larger than $1 / 2$. The states are symmetrical about the center of the largest incoherent cluster. Figure 1(D) shows a $\mathrm{C} 2 \mathrm{n}+1 \mathrm{P} 1$ chimera state with $\mathrm{n}=2$ at $\kappa_{1}=4$ and $\kappa_{2}=1.4$.

\subsection{Stability regimes of typical chimera states}

We first explore the stability regimes of the typical chimera states in the plane of $\kappa_{1}$ and $\kappa_{2}$. To do it, we consider two transition diagrams with $\kappa_{2}$, forward and backward continuations. The coupling range $\kappa_{2}$ is successively increased/decreased by a $\delta \kappa_{2}$ in the forward/backward continuation and the initial conditions for one $\kappa_{2}$ are the final state of the previous one. During the forward/backward continuation, the model evolves about $10^{3}$ time units for each $\kappa_{2}$ to ensure that the steady state is reached. When the transition such as the one between $\mathrm{C} 2 \mathrm{P} 1$ states and $\mathrm{C} 4 \mathrm{P} 1$ states is taken into consideration, we first build a $\mathrm{C} 2 \mathrm{P} 1$ state or a $\mathrm{C} 4 \mathrm{P} 1$ state with random initial conditions and, then, start the fowrward/backward continuation using the developed $\mathrm{C} 2 \mathrm{P} 1 / \mathrm{C} 4 \mathrm{P} 1$ state as the initial conditions. We monitor the quantity $\sigma(x)=\left\langle\left[\dot{\theta}(x)-\omega_{e}(x)\right]^{2}\right\rangle_{t}$. Generally, $\sigma(x)$ is zero in the coherent clusters for stationary chimera states [48], which can be used as a criterion distinguishing chimera states with different numbers of coherent clusters. Figures 3 (a) and (b) show the two transition diagrams between $\mathrm{C} 2 \mathrm{P} 1$ and $\mathrm{C} 4 \mathrm{P} 1$ states at $\kappa_{1}=4$. Clearly, the transition between $\mathrm{C} 2 \mathrm{P} 1$ and $\mathrm{C} 4 \mathrm{P} 1$ states is not continuous. When $\kappa_{2}$ increases from 0 to 0.5 , the $\mathrm{C} 2 \mathrm{P} 1$ state first evolves into a C1P1 state in which the smaller coherent cluster in the $\mathrm{C} 2 \mathrm{P} 1$ one is lost, and then into a state in which all oscillators become desynchronized. The C4P1 state shows up suddenly when $\kappa_{2}$ is beyond $\kappa_{2}=2.1$. As an illustration of chimera dynamics, the snapshots $\theta(x)$ at different 


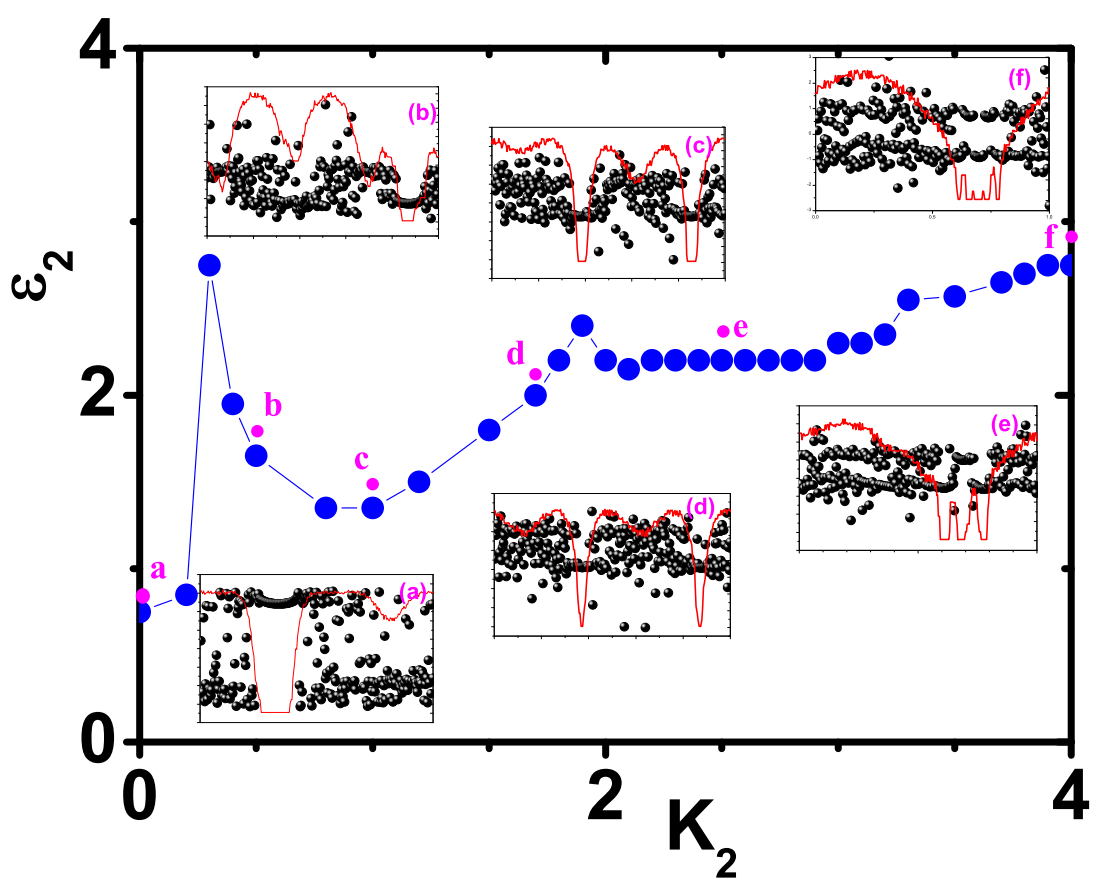

Figure 5. (color online)The critical $\epsilon_{2}$ is plotted against $\kappa_{2}$. Above the curve, the chimera states with the characteristics resulted from the biharmonic interaction are born. Insets (a)-(f) show the snapshots $\theta(x)$ (black dots) and the effective frequencies $\omega_{e}(x)$ (red curves) at parameter sets denoted by magenta dots. (a) $\kappa_{2}=0$ and $\epsilon_{2}=0.8$; (b) $\kappa_{2}=0.5$ and $\epsilon_{2}=1.73$; (c) $\kappa_{2}=1$ and $\epsilon_{2}=1.5$; (d) $\kappa_{2}=1.7$ and $\epsilon_{2}=2.2$; (e) $\kappa_{2}=2.5$ and $\epsilon_{2}=2.4$; (f) $\kappa_{2}=4$ and $\epsilon_{2}=2.9 . \kappa_{1}=4$ and $N=256$.

$\kappa_{2}$ are displayed in Figs. 3(c)-(e). On the other hand, the backward continuation shows that the C4P1 state persists till a lower $\kappa_{2}$ at around 1.5 and, then, the C1P1 state pops up abruptly which evolves into a C2P1 state gradually. Figures $3(\mathrm{f})$ and (g) show the transition diagrams between $\mathrm{C} 4 \mathrm{P} 1$ and $\mathrm{C} 8 \mathrm{P} 1$ states at $\kappa_{1}=4$. As shown in the figures, the transition between $\mathrm{C} 4 \mathrm{P} 1$ and $\mathrm{C} 8 \mathrm{P} 1$ is also discontinuous and there exist C2P1 chimera states with spatial period $1 / 2$ between them. The snapshots of these different chimera states are presented in Figs. 3(h)-(j).

Basing on the transition diagrams at different $\kappa_{1}$, we have the stability regimes in the plane of $\kappa_{1}$ and $\kappa_{2}$ for typical chimera states in Fig. 4. The C2P1 state is always stable at $\kappa_{2}=0$ provided that $\kappa_{1}>0.06$. The regime for the $\mathrm{C} 2 \mathrm{P} 1$ state is confined to a narrow regime around $\kappa_{2}=0$ with a threshold at around $\kappa_{2}=0.05$. The stability regime of the $\mathrm{C} 4 \mathrm{P} 1$ state, which is enclosed by the blue curves in Fig. 4 , is separated from that of the $\mathrm{C} 2 \mathrm{P} 1$ state by the chimera state $\mathrm{C} 1 \mathrm{P} 1$. Interestingly, we find that the stabilities of the $\mathrm{C} 2 \mathrm{nP} 1$ states with the spatial period $1 / n$ strongly depend on $\kappa_{2}$ but are not sensitive to $\kappa_{1}$. On the other hand, the stabilities of the C1P2 and C2P2 chimera states are sensitive to $\kappa_{1}$. As shown, the C1P2 chimera state requires large $\kappa_{1}$ 




Figure 6. (color online) (A) The solutions $\Delta \theta$ to Eq. (7) are plotted against $p$ where $\Delta \theta=0$ and $\Delta \theta=\pi$ are unstable. (B) The effects of $\kappa_{1}$ on chimera dynamics at $\kappa_{2}=0$. (B1)-(B3) The snapshots $\theta(x)$ at $\kappa_{1}=0.04, \kappa_{1}=0.07$ and $\kappa_{1}=0.5$, respectively. (B4) The sizes of two coherent clusters in the $\mathrm{C} 2 \mathrm{P} 1$ states are plotted against $\kappa_{1}$. (B5) The maximum (in red) and the minimum (in black) in the graph $\left|\omega_{e}(x)\right|$ are plotted against $\kappa_{1}$. (C) The effects of $\kappa_{2}$ on chimera dynamics at $\kappa_{1}=0$. (C1)-(C4) The snapshots $\theta(x)$ at $\kappa_{2}=0.1, \kappa_{2}=0.2, \kappa_{2}=0.8$, and $\kappa_{2}=2$, respectively. (C5) The maximum (in red) and the minimum (in black) in the graph $\left|\omega_{e}(x)\right|$ are plotted against $\kappa_{2} . \mathrm{N}=256$.

while the $\mathrm{C} 2 \mathrm{P} 2$ one prefers intermediate $\kappa_{1}$. Even if only a few types of chimera states are taken into considerations, the overlap amongst the stability regimes is apparent in Fig. 4, which suggests that the coexistence among different types of chimera states is prevailing in the model Eq. (2)).

As mentioned in the model section, to realize the chimera states with the features resulting from the biharmonic interaction, the strength of the second harmonic interaction has to be strong enough. Then, we consider chimera dynamics in the plane of $\kappa_{2}$ and $\epsilon_{2}$. Instead of the stability regimes of different chimera states, we concern with the onset of the chimera states observed above with the change of $\epsilon_{2}$. For a given $\kappa_{2}$, we simulate the model Eq. (2) with random initial conditions at different $\epsilon_{2}$. For each $\epsilon_{2}$, we perform simulations tens of times. If no chimera state can be developed, $\epsilon_{2}$ is below the critical value. Following this way, we locate the critical $\epsilon_{2}$ for different $\kappa_{2}$ and the curve of the critical $\epsilon_{2}$ against $\kappa_{2}$ is presented in Fig. 15. The critical $\epsilon_{2}$ does not change monotonically with $\kappa_{2}$. At around $\kappa_{2}=0.5$, the critical $\epsilon_{2}$ jumps to a high value. In the range of $\kappa_{2} \in(0.5,2)$, the curve behaves like a parabolic one. Furthermore, the critical $\epsilon_{2}$ increases with $\kappa_{2}$ when $\kappa_{2}>2$. Interestingly, in these extensive simulations, we find that, with the increase of $\epsilon_{2}$ from the critical value, $C n P 1$ chimera states with spatial period $1 / n$ are born firstly for $\kappa_{2}<2$ while multi-cluster chimera states with reflection symmetry are born firstly for $\kappa_{2}>2$. Some examples of chimera states at the parameters just above the critical curves are presented in the insets in Fig. 5 . 


\subsection{The link between chimera states and synchronization in globally coupled model}

To better understand the above chimera dynamics, it is helpful to investigate the synchronous dynamics in globally coupled oscillators $\left(\kappa_{1}=0\right.$ and $\left.\kappa_{2}=0\right)$. In such a synchronous state, oscillators will split into two groups and, in each of them, oscillators hold a same phase. Denoting $\theta_{i}(i=1,2)$ as the phases held by oscillators in these two groups, we have

$$
\begin{aligned}
\Omega= & -\epsilon_{1} R_{1} \sin \left(\theta_{i}-\Theta_{1}+\alpha_{1}\right) \\
& -\epsilon_{2} R_{2} \sin 2\left(\theta_{i}-\Theta_{2}+\alpha_{2}\right), i=1,2
\end{aligned}
$$

with $\Omega=d \theta_{1} / d t=d \theta_{2} / d t$. Suppose that the fraction of oscillators taking $\theta_{1}$ is $p$. Then substituting the order parameters Eq. (3) into Eq. (7), we have the relationship between $\Delta \theta=\theta_{2}-\theta_{1}$ and $p$, which is shown in Fig. 6(A) (A relevant theoretical work has been done in the reference [49]). At a given $p$, there are four different $\Delta \theta$ satisfying Eq. (7) in which $\Delta \theta=0$ and $\Delta \theta \simeq \pi$ are unstable. The other two $\Delta \theta$ are stable: $\Delta \theta \simeq \pi / 2$ and $\Delta \theta \simeq-\pi / 2$ (or $\Delta \theta \simeq 3 \pi / 2$ ). Interestingly, the values of these two stable $\Delta \theta$ are close to both the phase difference between the two groups in coherent clusters for C1P2 and $\mathrm{C} 2 \mathrm{P} 2$ chimeras and the phase difference between adjacent coherent clusters for $\mathrm{C} 2 \mathrm{nP} 1$ chimeras with spatial period $1 / n$.

To elucidate the relation between the synchronous dynamics in the case of global coupling and the chimera dynamics in the case of nonlocal coupling and to explore the impacts of $\kappa_{1}$ and $\kappa_{2}$ on chimera dynamics, we investigate the model Eq. (2) by increasing either $\kappa_{1}$ or $\kappa_{2}$ from zero. Figures 6(B1) and (B2) show the snapshots $\theta(x)$ for two nonzero $\kappa_{1}$ at $\kappa_{2}=0$. At small $\kappa_{1}$, the synchronous dynamics persists. Beyond a critical $\kappa_{1}$ which depends on $p$, the synchronous state becomes unstable and oscillators self-organize themselves into a $\mathrm{C} 2 \mathrm{P} 1$ chimera state during which the phase difference between oscillators in synchronization keeps almost unchanged. Further increasing $\kappa_{1}$ does not alter the nature of the $\mathrm{C} 2 \mathrm{P} 1$ state [see Fig. 6(B3)]. Figure 6(B4) shows the sizes of coherent clusters $S$ against $\kappa_{1}$. The sizes of both coherent clusters decrease with $\kappa_{1}$ monotonically. On the other hand, the size difference between two coherent clusters seems to be independent of $\kappa_{1}$. We also monitor the maximum and the minimum in the graph $\left|\omega_{e}(x)\right|$. The results presented in Fig. 6(B5) show that the decrease of the fraction of coherent oscillators with $\kappa_{1}$ is accompanied by the fall of $\left|\omega_{e}(x)\right|$. In contrast, $\kappa_{2}$ displays quite different impacts on the model dynamics. Figures 6(C1)-(C3) suggest that increasing $\kappa_{2}$ leads to more coherent clusters. At sufficient large $\kappa_{2}$, the states with many coherent clusters are replaced by irregular dynamics [see Fig. 6(C4)]. Figure 6)(C5) shows that the maximum and the minimum in the graph $\left|\omega_{e}(x)\right|$ changes prominently only for irregular dynamics.

In short, the results in Fig. 6] suggest that the synchronous dynamics in globally coupled phase oscillators is responsible for the $\pi / 2$ phenomenon for both the phase difference between two groups of coherent oscillators in C1P2 and C2P2 chimera states and the phase difference between adjacent coherent clusters in chimera states such as $\mathrm{C} 2 \mathrm{nP} 1$ ones. Figure 6 also suggests that it is the coupling range $\kappa_{2}$ but not the coupling 
range $\kappa_{1}$ to determine the number of coherent clusters in $\mathrm{CnP} 1$ chimera states, which is in agreement with Fig. 4. In addition, Fig. 6 shows that synchronous dynamics in the model Eq. (2) are always unstable provided that $\kappa_{1}$ is not close to zero. Without the competition with the synchronous dynamics, chimera states in the presence of the biharmonic interaction can always be realized with arbitrary initial conditions, which is quite different from the chimera states in the absence of the second harmonic interaction.

\section{Conclusion}

In conclusion, we have investigated the nonlocally coupled phase oscillators with the biharmonic interaction. We found chimera states with peculiar characteristics resulting from the interplay between the first and second harmonic interactions. In C1P2 and C2P2 chimera states, oscillators in the same coherent cluster spilt into two groups with the phase difference between them at around $\pi / 2$. In $\mathrm{C} 2 \mathrm{nP} 1$ chimera states with spatial period $1 / n$, the phase difference between adjacent coherent clusters is not $\pi$ but around $\pi / 2$. We also found the prevalence of multi-cluster chimera states. Multi-cluster chimera states have been found in many systems with kernel function taking the form of step function [23, 46, 24]. In those works, reducing the coupling range favors the appearance of more coherent clusters. However, for kernel function taking the exponential one, only transient multi-cluster chimera states are observed for phase oscillators [47]. Therefore, multi-cluster chimera states with more than 2 coherent clusters are not trivial for nonlocally coupled phase oscillators. Furthermore, we found that the coupling range $\kappa_{1}$ has little influence on the number of coherent clusters. In contrast, increasing the coupling range $\kappa_{2}$ seems to produce more coherent clusters when $\kappa_{2}$ is not very large.

\section{Acknowledge}

This work was supported by NSF of China under Grants Nos. 11575036, 71301012, and 11505016.

\section{References}

[1] Kuramoto Y, Battogtkh D. Coexistence of coherence and incoherence in nonlocally coupled phase oscillators. Nonlinear Phenom Complex Syst 2002;5:380.

[2] Abrams D M, Strogatz S H. Chimera states for coupled oscillators. Phys Rev Lett 2004;93:174102.

[3] Abrams D M, Mirollo R, Strogatz S H, Wiley D A. Solvable model for chimera states of coupled oscillators. Phys Rev Lett 2008;101:084103.

[4] Laing C R. The dynamics of chimera states in heterogeneous Kuramoto networks. Physica D 2009;238:1569.

[5] Motter A E. Nonlinear dynamics: Spontaneous synchrony breaking. Nat Phys 2010;6:164.

[6] Nkomo S, Tinsley M R, Showalter K. Chimera States in populations of nonlocally coupled chemical oscillators. Phys Rev Lett 2013;110:244102.

[7] Sethia G C, Sen A. Chimera states: The existence criteria revisited. Phys Rev Lett 2014;112:144101.

[8] Zakharova A, Kapeller M, Schöll E. Chimera death: Symmetry breaking in dynamical networks. Phys Rev Lett 2014;112:154101. 
[9] Banerjee T. Mean-field-diffusion-induced chimera death state. EPL 2015;110:60003.

[10] Pazó D, Montbrió E. Low-dimensional dynamics of populations of pulse-Coupled oscillators. Phys Rev X 2014;4:011009.

[11] Clerc M G, Coulibaly S, Ferré M A, García-Ñustes M A, Rojas R G. Chimera-type states induced by local coupling. Phys Rev E 2016;93:052204.

[12] Laing C R. Chimeras in networks with purely local coupling. Phys Rev E 2015;92:050904(R).

[13] Sheeba J H, Chandrasekar V K, Lakshmanan M. Globally clustered chimera states in delay-coupled populations. Phys Rev E 2009;79:055203(R).

[14] Yeldesbay A, Pikovsky A, Rosenblum M. Chimeralike States in an Ensemble of Globally Coupled Oscillators. Phys Rev Lett 2014;112:144103.

[15] Wolfrum M, Omel'chenko O E. Chimera states are chaotic transients. Physical Review E 2011;84(1):15201.

[16] Martens E A, Laing C R, Strogatz S H. Solvable model of spiral wave chimeras. Phys Rev Lett 2010;104:044101.

[17] Gu C, St-Yves G, Davidsen J. Spiral wave chimeras in complex oscillatory and chaotic systems. Phys Rev Lett 2013;111:134101.

[18] Li B, Dierckx H. Spiral wave chimeras in locally coupled oscillator systems. Phys Rev E 2016;93:020202(R).

[19] Panaggio M J, Abrams D M. Chimera states on a flat torus. Phys Rev Lett 2013;110:094102.

[20] Zhu Y, Zheng Z, Yang J. Chimera states on complex networks. Phys Rev E 2014;89:022914.

[21] Omelchenko I, Maistrenko Y, Hövel P, Schöll E. Loss of coherence in dynamical networks: Spatial chaos and chimera states. Phys Rev Lett 2011;106:234102.

[22] Omelchenko I, Riemenschneider B, Hövel P, Maistrenko Y, Schöll E. Transition from spatial coherence to incoherence in coupled chaotic systems. Phys Rev E 2012;85:026212.

[23] Omelchenko I, Omelchenko O E, Hövel P, Schöll E. When nonlocal coupling between oscillators becomes stronger: Patched synchrony or multichimera states. Phys Rev Lett 2013;110:224101.

[24] Hizanidis J, Kanas V, Bezerianos A, Bountis T. Chimera states in networks of nonlocally coupled Hindmarsh-Rose neuron models. Int J Bifurcat Chaos 2014;24:1450030.

[25] Bera B K, Ghosh D, Lakshmanan M. Chimera states in bursting neurons. Phys Rev E 2016;93:012205.

[26] Hagerstrom A M, Murphy T E, Roy R, Hövel P, Omelchenko I, Schöll E. Experimental observation of chimeras in coupled-map lattices. Nat Phys 2012;8:658.

[27] Viktorov E A, Habruseva T, Krischer K, García-Morales V. Coherence and incoherence in an optical comb. Phys Rev Lett 2014;112:224101.

[28] Tinsley M R, Nkomo S, Showalter K. Chimera and phase cluster states in populations of coupled chemical oscillators. Nat Phys 2012;8:662.

[29] Schmidt L, Schönleber K, Krischer K, García-Morales V. Coexistence of synchrony and incoherence in oscillatory media under nonlinear global coupling. Chaos 2014;24:013102.

[30] Martens E A, Thutupalli S, Fourriere A, Hallatschek O. Chimera states in mechanical oscillator networks. Proc Nat Acad Sci(USA) 2013;110:10563.

[31] Kapitaniak T, Kuzma P, Wojewoda J, Czolczynski K, Maistrenko Y. Imperfect chimera states for coupled pendula. Sci Rep 2014;4:6379.

[32] Olmi S, Martens E A, Thutupalli S, Torcini A. Intermittent chaotic chimeras for coupled rotators. Phys Rev E 2015;92:030901(R).

[33] Ermentrout G B, Kopell N. Multiple pulse interactions and averaging in systems of coupled neural oscillators. J Math Biol 1991;29:1995.

[34] Izhikevich E M. Dynamical systems in neuroscience: The geometry of excitability and bursting. The MIT press 2007.

[35] Czolczyński K, Perlikowski P, Stefański A, Kapitaniak T. Synchronization of the self-excited pendula suspended on the vertically displacing beam. Commun Nonlinear Sci Numer Simul $2013 ; 18: 386$. 
[36] Goldobin E, Kleiner R, Koelle D, Mints R G. Phase Retrapping in a Pointlike $\phi$ Josephson Junction: The Butterfly Effect. Phys Rev Lett 2013;111:057004.

[37] Komarov M, Pikovsky A. Multiplicity of Singular Synchronous States in the Kuramoto Model of Coupled Oscillators. Phys Rev Lett 2013;111:204101.

[38] Li K, Ma S, Li H, Yang J. Transition to synchronization in Kuramoto model with the first and the second order interaction terms. Phys Rev E 2014;89:032917.

[39] Daido H. Generic scaling at the onset of macroscopic mutual entrainment in limit-cycle oscillators with uniform all-to-all coupling. Phys Rev Lett 1994;73:760.

[40] Daido H. Onset of cooperative entrainment in limit-cycle oscillators with uniform all-to-all interactions: bifurcation of the order function. Physica D 1996;91:24.

[41] Suda Y, Okuda K. Persistent chimera states in nonlocally coupled phase oscillators. Phys Rev E 2015;92:060901(R).

[42] Ashwin P, Burylko O. Weak chimeras in minimal networks of coupled phase oscillators. Chaos 2015;25:13106.

[43] Bick C, Ashwin P. Chaotic weak chimeras and their persistence in coupled populations of phase oscillators. Nonlinearity 2016;29:1468.

[44] Bick C. Isotropy of Angular Frequencies and Weak Chimeras With Broken Symmetry. arXiv:2016;1512:01321.

[45] Sethia G C, Sen A, Atay F M. Clustered chimera states in delay-coupled oscillator systems. Phys Rev Lett 2008;100:144102.

[46] Omelchenko I, Provata A, Hizanidis J, Hövel P, Schöll E. Robustness of chimera states for coupled FitzHugh-Nagumo oscillators. Phys Rev E 2015;91:022917.

[47] Maistrenko Y L, Vasylenko A, Sudakov O, Levchenko R, Maistrenko V L. Cascades of multiheaded chimera states for coupled phase oscillators. Int J Bifurcation Chaos 2014;24:1440014.

[48] Zhu Y, Li Y, Zhang M, Yang J. The oscillating two-cluster chimera state in non-locally coupled phase oscillators. EPL 2012;97:10009.

[49] Orosz G, Moehlis J, Ashwin P. Designing the Dynamics of Globally Coupled Oscillators. Prog Theor Phys 2009;122:611. 